\title{
Glucose control during Ramadan fasting in a teenager with type 1 diabetes on MiniMed 670G hybrid closed-loop system
}

\author{
Goran Petrovski $^{1}$ (D) $\cdot$ Fawziya Al Khalaf ${ }^{1} \cdot$ Judith Campbell $^{1} \cdot$ Khalid Hussain $^{1} \cdot$ Hannah Fisher $^{1} \cdot$ Fareeda Umer $^{1}$
}

Received: 1 July 2019 / Accepted: 22 August 2019/ Published online: 31 August 2019

(C) The Author(s) 2019

\section{Introduction}

Fasting during the month of Ramadan includes abstinence from drink and food from dawn till sunset and can be from several hours to more than $20 \mathrm{~h}$ per day. Prepubertal children and people with acute or chronic medical conditions are excused from fasting, which may aggravate their condition [1]. Children with type 1 diabetes (T1D) are considered a high-risk population, and it is recommended not to fast during Ramadan. However, a significant number of patients prefer to fast to respect their religion.

One of the treatment's options for T1D is the MiniMed 670G hybrid closed-loop (HCL) system (Medtronic, USA), which uses an algorithm capable of automatically adjusting basal insulin delivery in response to glucose sensor readings transmitted to the insulin pump every $5 \mathrm{~min}$.

We present the case with T1D where the glucose control was managed using the MiniMed 670G HCL system during the month of the Ramadan. To the best of our knowledge, this is the first patient to be reported on fasting during Ramadan and HCL system.

\section{Case presentation}

A 13-year-old male patient with a 4-year history of T1D using MiniMed 670G HCL system was fasting for the first time around $14 \mathrm{~h}$ per day for the month of Ramadan. Patient had HbA1c levels between 8.2 and $11.8 \%$ (66-105 mmol/ mol) with previous treatment (multiple daily injections with

Managed By Massimo Porta.

Goran Petrovski

gpetrovski@sidra.org

1 Division of Endocrinology and Diabetes, Department of Pediatric Medicine, Sidra Medicine, HB 6E 219, Al Luqta Street, Education City North Campus, PO Box 26999, Doha, Qatar self-monitoring of blood glucose). MiniMed 670G HCL system was initiated 5 months before the study, and $\mathrm{HbA} 1 \mathrm{c}$ of $6.6 \%(49 \mathrm{mmol} / \mathrm{mol})$ and time in range $(70-180 \mathrm{mg} /$ $\mathrm{dl} ; 3.9-10.0 \mathrm{mmol} / \mathrm{l})$ of $76 \%$ ) were achieved. The patient uploaded the HCL system on Carelink Personal Software on days 1, 14 and 30 during the Ramadan, and consultation was given by phone. Glucose and insulin metrics were analyzed 1 month before and during Ramadan period.

Patient broke the fast twice in the afternoon period during the first week of Ramadan due to a mild hypoglycemic event. We advised him to use temporary target for $2-4 \mathrm{~h}$ if glucose levels reached $80 \mathrm{mg} / \mathrm{dl}(4.4 \mathrm{mmol} / \mathrm{l})$ to avoid further glucose decrease.

Despite correct carbohydrate counting, a slight increase in glucose values (18-00 h) was noted due to breaking the daily fast with eating at evening meal, iftar (as shown in Fig. 1). We recommended to increase the meal bolus by $10-20 \%$, if the meal contained more than $100 \mathrm{~g}$ (e.g., to increase the bolus by $20 \%$ when $110 \mathrm{~g}$ of carbohydrates were eaten, $132 \mathrm{~g}$ of carbohydrates was entered into the bolus wizard calculator) and to split bolus insulin 40-50\% before and $50-60 \%$ after the meal, as the "dual wave" and "square" boluses are disabled in MiniMed 670G HCL system.

Average auto-basal/basal insulin amount per day (as shown in Fig. 1) was significantly lower in the period 4 am-6 pm in fasting hours during Ramadan compared to the same period in the non-fasting hours before Ramadan $(p<0.05)$.

We did not find any significant difference in time spent in AM, AM exits, sensor wear and total daily insulin per day before and during Ramadan (as shown in Fig. 1). No diabetic ketoacidosis and severe hypoglycemia were detected during and before Ramadan. 


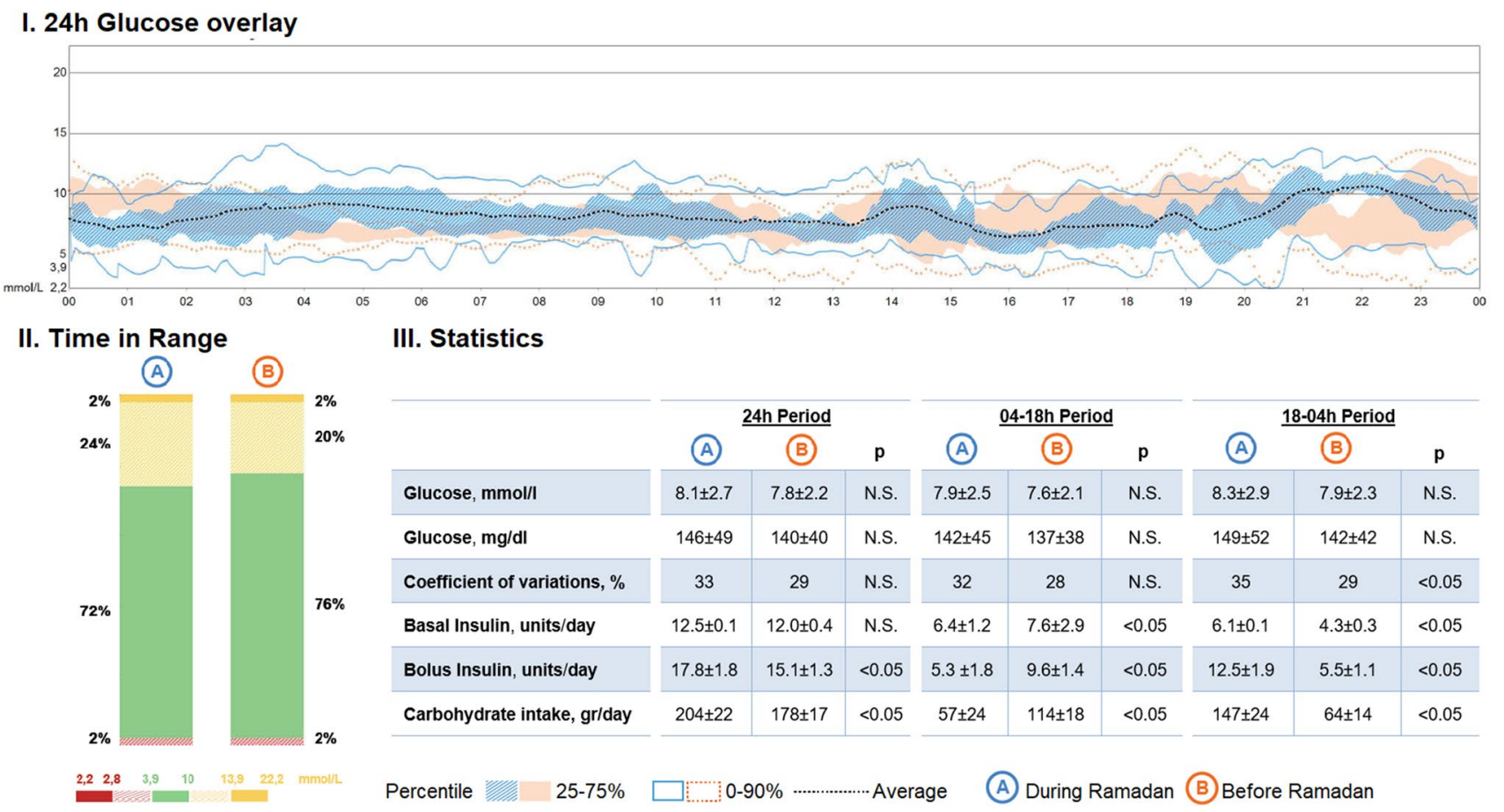

Fig. 1 Glucose control during and before Ramadan. I. 24-h glucose overlay, percentile comparison of two periods; blue color, one month during Ramadan; orange color, one month before Ramadan. II. Time in range, glucose distribution comparison. III. Statistics, glucose and

\section{Discussion and conclusion}

Despite certain limitations, we have provided a general overview on successful glucose control in child with T1D using the MiniMed 670G HCL system during the month of Ramadan.

Our case presented satisfactory glucose control without significant change in time in ranges, without influencing percentage of time spent with low glucose values 1 month during and 1 month before Ramadan. Like our findings, insulin pump can significantly reduce hypoglycemic events without increasing $\mathrm{HbA1c}$ values [2].

Insulin pumps and multiple daily injections are comparable in relation to glycemic control during Ramadan fasting in patients with T1D, where healthcare professionals are required to provide Ramadan-specific diabetes care. In our case, we did not provide specific diabetes care before Ramadan, which shows simple management of HCL system in T1D during Ramadan fasting.

Our case shows minimal adjustment of the HCL system before and during Ramadan with using a temporary target in the afternoon and increasing the meal bolus by $10-20 \%$ for huge meals in the evening, without any change in basal and bolus insulin settings. Our results differ to recently published studies on open-loop CSII, insulin metrics comparison. Glucose, basal insulin, bolus insulin and carbohydrates are expressed as mean plus SD for selected periods: $24 \mathrm{~h}, 04-18 \mathrm{~h}$ and 18-04 h. A-period, 1 month during Ramadan; B-period, 1 month before Ramadan (color figure online)

which have shown additional time in counseling on dietary modifications and therapeutic changes [3]. Automatically basal adjustments with MiniMed 670G are more practical during Ramadan compared to the clinical guidelines, where the reduction in basal rate by $5-50 \%$ for fasting days and weekly reassessment for further adjustments are recommended [4].

Like other authors, we did not find any diabetic ketoacidosis and severe hypoglycemia during the month of Ramadan [5].

HCL system with automatic adjustments of basal insulin delivery in response to CGM readings, temporary target feature, good collaboration between health providers and patient allows satisfactory glucose control during fasting of $14 \mathrm{~h}$ per day during the month of Ramadan, which we believe would not have been achieved using multiple daily insulin regimen or CSII. Further clinical trials should be performed to confirm our findings.

Acknowledgements Open Access funding provided by the Qatar National Library. The authors thank the patient and his family for their collaboration during the study period.

\section{Compliance with ethical standards}

Conflict of interest The authors declare that they have no conflict of interest. 
Ethical approval All procedures performed in studies involving human participants were in accordance with the ethical standards of the institutional and/or national research committee and with the 1964 Helsinki declaration and its later amendments or comparable ethical standards.

Informed consent Informed consent was obtained from the patient.

Open Access This article is distributed under the terms of the Creative Commons Attribution 4.0 International License (http://creativeco mmons.org/licenses/by/4.0/), which permits unrestricted use, distribution, and reproduction in any medium, provided you give appropriate credit to the original author(s) and the source, provide a link to the Creative Commons license, and indicate if changes were made.

\section{References}

1. Abolaban H, Al-Moujahed A (2017) Muslim patients in Ramadan: a review for primary care physicians. Avicenna J Med 7(3):81-87
2. Elbarbary NS (2016) Effectiveness of the low-glucose suspend feature of insulin pump during fasting during Ramadan in type 1 diabetes mellitus. Diabetes Metab Res Rev 32:623-633

3. Afandi B, Kaplan W, Al Hassani N et al (2017) Correlation between pre-Ramadan glycemic control and subsequent glucose fluctuation during fasting in adolescents with type 1 diabetes. $\mathbf{J}$ Endocrinol Invest 40:741-744

4. Bajaj HS, Abouhassan T, Ahsan MR et al (2019) Diabetes Canada position statement for people with types 1 and 2 diabetes who fast during Ramadan. Can J Diabetes 43:3-12

5. Gad H, Al-Muhannadi H, Mussleman P et al (2019) Continuous subcutaneous insulin infusion versus multiple daily insulin injections in patients with type 1 diabetes mellitus who fast during Ramadan: a systematic review and meta-analysis. Diabetes Res Clin Pract 151:265-274

Publisher's Note Springer Nature remains neutral with regard to jurisdictional claims in published maps and institutional affiliations. 